\title{
Speckle masking interferometry with the Large Binocular Telescope
}

\author{
T. Reinheimer, K.-H. Hofmann, M. Schöller, and G. Weigelt \\ Max-Planck-Institut für Radioastronomie, Auf dem Hügel 69, 53121 Bonn, Germany \\ tr@specklec.mpifr-bonn.mpg.de, khh@specklec.mpifr-bonn.mpg.de, ms@specklec.mpifr-bonn.mpg.de, \\ weigelt@mpifr-bonn.mpg.de
}

Received January 18; accepted March 17, 1996

\begin{abstract}
We present a method for interferometric imaging with the Large Binocular Telescope (LBT) at optical and infrared wavelengths. For example, at $\lambda=550 \mathrm{~nm}$ a resolution of 6.1 mas can be obtained. The $u v$-coverage is excellent due to the small distance between the two $8.4 \mathrm{~m}$ mirrors. We show laboratory and computer experiments of LBT speckle masking interferometry. The raw data were produced by simulating light propagation in the atmosphere, the LBT pupil function, earth rotation, and photon noise. The generated data sets consist of up to 200000 LBT interferograms per experiment with 200 to 2000 photoevents per interferogram. 200000 interferograms correspond to only 1.1 hours observing time for a frame rate of 50 frames/sec. In the computer simulations a Fried parameter of $40 \mathrm{~cm}$ was simulated which corresponds to 0.35 arcsec seeing. Diffractionlimited images were reconstructed from the various data sets by a modified version of the speckle masking method (bispectral analysis, triple correlation method) and the iterative building block method. The reconstructed images show the dependence of the signal-to-noise ratio on photon noise and other parameters. In one of the experiments the object was a compact cluster of four stars and the interferograms consisted of only 200 photoevents per interferogram. 200 photoevents per interferogram correspond to a total $V$ magnitude $\sim 14.3$ for two $8 \mathrm{~m}$ telescopes, $20 \mathrm{msec}$ exposure time per interferogram, $5 \mathrm{~nm}$ filter bandwidth, and $10 \%$ quantum efficiency of detector plus optics. In this experiment the magnitudes of the four individual stars were 15.6, 15.8, 16.4, and 17.1. In a second experiment a compact galaxy with total magnitude of 11.3 and magnitude $\sim 14$ of the faintest resolution element was simulated and a diffraction-limited image reconstructed successfully from only 200000 interferograms (1.1 hour observing time). Objects of about 18th magnitude can be observed if observing time is increased and observations are made simultaneously in many spectral channels. An
\end{abstract}

Send offprint requests to: T. Reinheimer advantage of speckle masking is that it can be applied to objects fainter than 14th $V$ magnitude, whereas for adaptive optics (with natural reference stars for wavefront sensing) the object or the reference star has to be brighter than about 14th magnitude. Diffraction-limited images of objects fainter than 18th magnitude can be obtained by LBT speckle masking observations if partial wavefront compensation (low-order adaptive optics) is achieved by an artificial laser guide star system (Foy \& Labeyrie 1985; Fugate et al. 1991; Primmerman et al. 1991).

Key words: instrumentation: interferometers methods: data analysis - techniques: image processing — techniques: interferometric — telescopes

\section{Introduction}

In a few years from now the LBT (Strittmatter 1988; McCarthy et al. 1988) will be available for optical interferometry. The LBT will consist of two $8.4 \mathrm{~m}$ telescopes on a common mount with the mirrors only $14.4 \mathrm{~m}$ apart from center to center. Therefore, excellent $u v$-coverage can be obtained. The largest baseline is $22.8 \mathrm{~m}$ and the angular resolution $1.22 \lambda / D$ of this interferometer is 6.1 milli-arcsec at $\lambda=550 \mathrm{~nm}$, i.e., $22.8 \mathrm{~m} / 2.4 \mathrm{~m}=9.5$ times higher than the resolution of the HST at the same wavelength.

At $\lambda \sim 550 \mathrm{~nm}$ the speckles in the LBT interferograms have a width of about $1.22 \lambda / D \sim 6.1$ mas and a length (corresponding to the $8.4 \mathrm{~m}$ mirror diameter) of about 16.5 mas. The number of speckles is approximately equal to the number of turbulence cells in front of the telescopes. For example, for a Fried parameter $r_{0}=40 \mathrm{~cm}(\sim 0.35$ arcsec seeing) and an $8 \mathrm{~m}$ class telescope, the number of speckles is about 400 per interferogram.

In this paper we present the theory and computer and laboratory simulations of interferometric speckle masking imaging with the LBT. Diffraction-limited images were 
reconstructed by a combination of a modified version of the speckle masking method (Weigelt 1977; Weigelt \& Wirnitzer 1983; Lohmann et al. 1983; Reinheimer \& Weigelt 1990; Reinheimer et al. 1993) and the building block method (Hofmann \& Weigelt 1990; Hofmann \& Weigelt 1993).
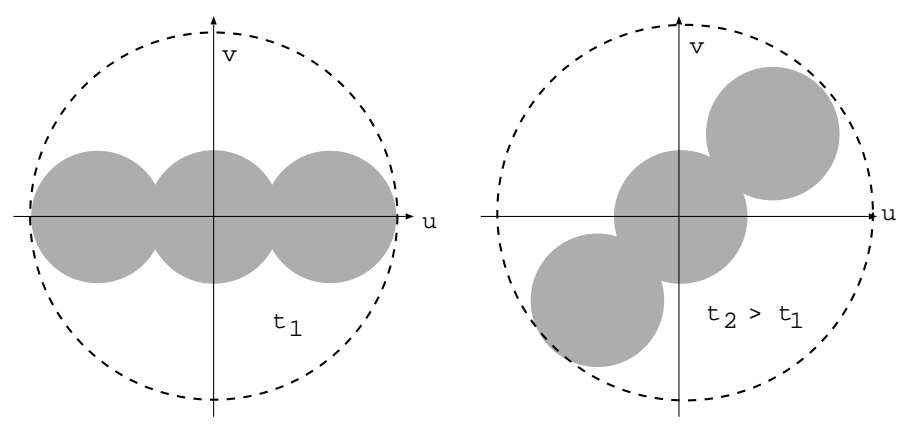

Fig. 1. Time dependence of the LBT $u v$-coverage $C\left(t_{i}\right)$ : the two shaded areas are the $u v$-coverages at two different times $t_{1}$ and $t_{2}$

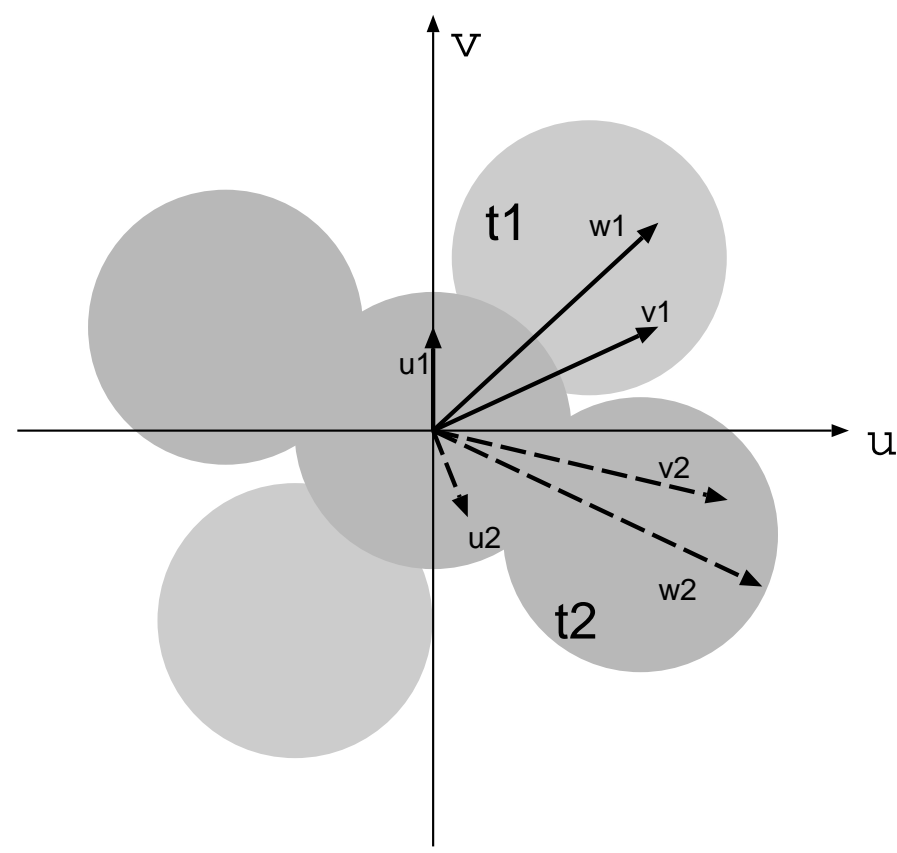

Fig. 2. Time dependence of the LBT $u v$-coverage and its influence on the bispectrum coverage (see text)

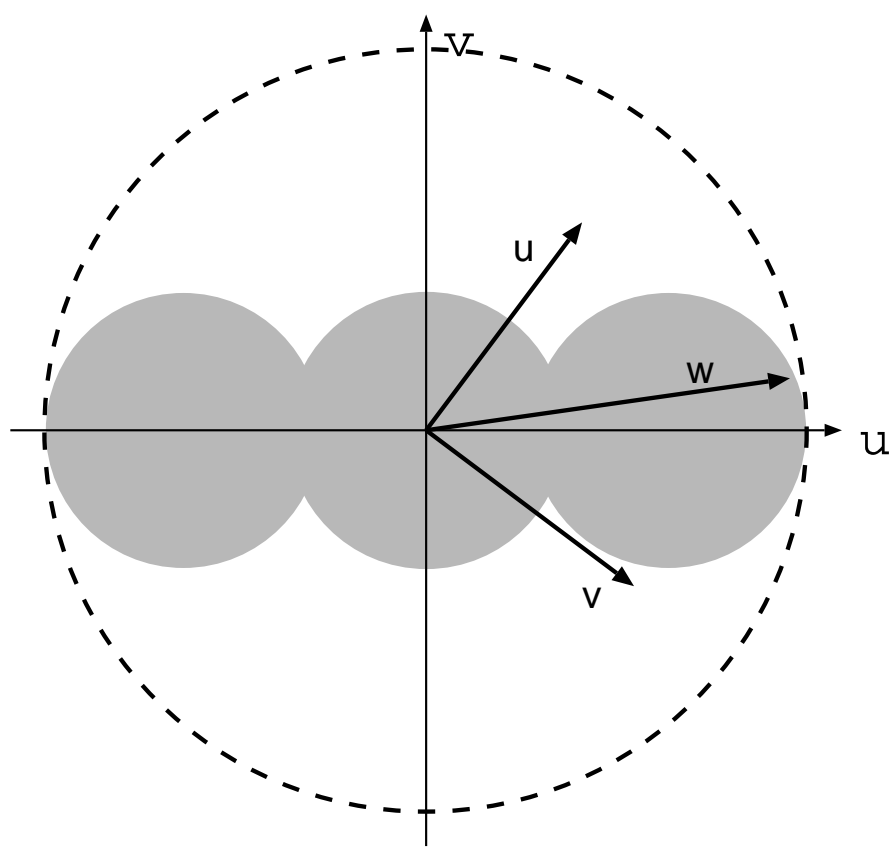

Fig. 3. Calculation of the bispectrum for complete and diluted $u v$-coverage. In the case of the LBT and the shown instantaneous $u v$-coverage, only bispectrum elements with $u$-vector, $v$-vector, and $w$-vector within the dark shaded area can be measured. For example, the bispectrum element corresponding to the shown vector triple cannot be measured during the shown LBT $u v$-coverage or other times, but it can be measured with a single-dish $22 \mathrm{~m}$ telescope.

\section{Image processing steps of the computer and laboratory experiments}

The instantaneous intensity distribution $i\left(x ; t_{i}\right)$ of the LBT interferograms can be described by the incoherent, space-invariant imaging equation

$i\left(x ; t_{i}\right)=\int o\left(x^{\prime}\right) p\left(x-x^{\prime} ; t_{i}\right) \mathrm{d} x^{\prime}$,

where $o(x)$ is the two-dimensional object intensity distribution, $p\left(x ; t_{i}\right)$ is the point spread function of the atmosphere/interferometer system, $x$ is a two-dimensional space vector. The time dependence $\left(t_{i}\right)$ is mentioned explicitly to point out the dependence of the psf and the optical transfer function (or $u v$-coverage) on the instantaneous projection of the interferometer pupil function (as seen from the object) in addition to the time dependence caused by the turbulent atmosphere (speckle boiling).

\subsection{Calculation of the average speckle masking bispectrum and aperture synthesis}

The first image processing step in speckle masking with normal single-dish telescopes (Weigelt 1977; Weigelt \& 
Wirnitzer 1983; Lohmann et al. 1983) is the calculation of the bispectrum

$I^{(3)}\left(u, v ; t_{i}\right)=I\left(u ; t_{i}\right) I\left(v ; t_{i}\right) I^{*}\left(u+v ; t_{i}\right)$

of each interferogram. $I$ denotes the Fourier transform of an interferogram, $u$ and $v$ are two-dimensional coordinate vectors in Fourier space, and the asterix denotes complex conjugation. For single-dish telescopes the time variable $t_{i}$ in Eq. (2) describes exclusively the time dependence caused by the turbulent atmosphere as the pupil function is time-invariant. In speckle imaging experiments with single-dish telescopes the object Fourier transform can be measured at all times at all spatial frequencies up to the diffraction cut-off frequency because of the perfect $u v$-coverage.

In speckle masking experiments with the LBT the conditions are different from single-dish telescopes because of the diluted pupil function. The instantaneous $u v$-coverage is not perfect, but it consists of an elongated structure of three circular regions extended up to the diffraction cutoff frequency of a single-dish $22 \mathrm{~m}$ telescope (see Fig. 1). The dashed circle shows the extension of the $u v$-coverage of a single-dish $22 \mathrm{~m}$ telescope, the shaded areas are the $u v$-coverage of the LBT at two different times. The $u v$ coverage changes with time since the astronomical object rotates relative to the LBT pupil function during the night. Therefore, in LBT interferometry, $u v$-coverage and bispectrum coverage are time-variant.

The Fourier transform $I\left(u ; t_{i}\right)$ of the instantaneous LBT interferogram $i\left(x ; t_{i}\right)$ recorded at time $t_{i}$ can be described by

$I\left(u ; t_{i}\right)=O(u) P\left(u ; t_{i}\right)$,

where $P\left(u ; t_{i}\right)$ is the optical transfer function of the atmosphere/interferometer system at time $t_{i}$ and $O(u)$ is the object Fourier transform. In LBT interferometry the time dependence of $P\left(u ; t_{i}\right)$ is caused (1) by the turbulent atmosphere and, additionally, (2) by the time dependence of the projected LBT interferometer pupil function as seen from the object (time dependence due to earth rotation). Each LBT interferogram yields information about the object structure only within the $u v$-coverage $C\left(t_{i}\right)$ which denotes the extension of the instantaneous transfer function $P\left(u ; t_{i}\right)$ (see Fig. 1). Therefore, the useful bispectrum $I_{C}^{(3)}\left(u, v ; t_{i}\right)$ of each LBT interferogram $i\left(x ; t_{i}\right)$ consists of only those factors $I\left(u ; t_{i}\right), I\left(v ; t_{i}\right)$ and $I^{*}\left(u+v ; t_{i}\right)$ with $u$-, $v$ - and $u+v$-vectors within the instantaneous $u v$-coverage $C\left(t_{i}\right)$ of the LBT transfer function $P\left(u ; t_{i}\right)$, i.e. we define

$$
\begin{aligned}
I_{C}^{(3)}\left(u, v ; t_{i}\right):= & I\left(u ; t_{i}\right) I\left(v ; t_{i}\right) I^{*}\left(u+v ; t_{i}\right), \\
& \text { with } u, v, u+v \in C\left(t_{i}\right) .
\end{aligned}
$$

Only bispectrum elements at those bispectrum coordinates $(u, v)$ with $u, v, u+v \in C\left(t_{i}\right)$ carry information about the source structure. The remaining bispectrum elements with at least one vector outside the actual $u v$ coverage $C\left(t_{i}\right)$ are zero-valued in the detector and photon noise-free case, however they introduce noise to the average bispectrum (integrated throughout the entire observing time) in the case of detector and photon noise. Therefore, it is important that these bispectrum elements are not used. Figure 2 shows examples of vector triples (e.g. $u_{1}, v_{1}$, and $w_{1}=u_{1}+v_{1}$ ) of bispectrum elements with and without information about the source structure at two different orientations of the pupil function relative to the object: for example, the bispectrum element $I^{(3)}\left(u_{1}, v_{1}\right)$ yields object information at recording time $t_{1}$, but it introduces noise at recording time $t_{2}$. Therefore, $I^{(3)}\left(u_{1}, v_{1}\right)$ has to be derived from measurements at time $t_{1}$, but not from measurements at $t_{2}$.

Due to the rotation of the object relative to the LBT pupil function, the $u v$-coverage of the LBT transfer function changes its orientation continuously by about $180^{\circ}$ within 12 hours observing time. Therefore, continuous observing of the object during one night creates the $u v$ coverage of a single-dish $22 \mathrm{~m}$ telescope (aperture synthesis by earth rotation). The ensemble average LBT interferometry bispectrum is the sum $\sum_{t_{i}} I_{C}^{(3)}\left(u, v ; t_{i}\right)$ over the aforementioned useful bispectra $I_{C}^{(3)}\left(u, v ; t_{i}\right)$ (Eq. 4) of the LBT interferograms $i\left(x ; t_{i}\right)$ recorded during the total observing time. Therefore, the four-dimensional bispectrum coverage is nearly perfect if observing time is about 12 hours. The bispectrum coverage is not completely filled in the sense that bispectrum elements with the largest $u$, $v$ - and $u+v$-vectors accessible with a $22 \mathrm{~m}$ single-dish telecope are not obtained with the diluted LBT aperture (see Fig. 3). But nevertheless all spatial frequencies up to the diffraction cut-off frequency of a $22 \mathrm{~m}$ single-dish telescope can be measured with the LBT.

\subsection{Compensation of photon bias terms}

The next image processing step is the compensation of the photon bias terms in the average LBT power spectrum $\sum_{t_{i}}\left|I_{C}\left(u ; t_{i}\right)\right|^{2}$ (Goodman 1985) and in the average LBT bispectrum $\sum_{t_{i}} I_{C}^{(3)}\left(u, v ; t_{i}\right)$ (Wirnitzer 1985; Pehlemann et al. 1992).

\subsection{Compensation of the speckle interferometry transfer function}

After compensation of the speckle interferometry transfer function (SITF) (Labeyrie 1970) in the photon biascompensated ensemble average LBT power spectrum, the object power spectrum $|O(u)|^{2}$ is obtained. Compensation is performed with the average LBT power spectrum of point source interferograms. In astronomical observations the SITF can be determined if the LBT interferometer is pointed alternately at the program object and a nearby 


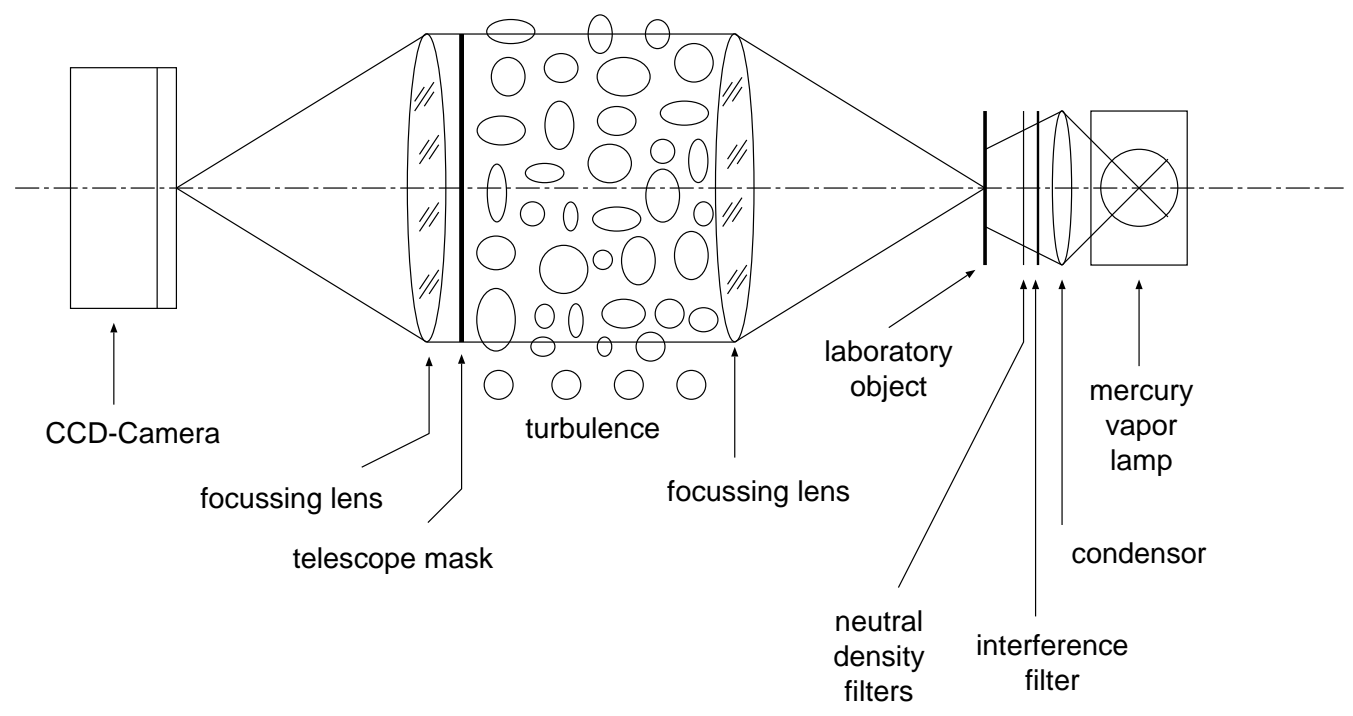

Fig. 4. Laboratory setup for simulating LBT interferograms

point source or if the object and the point source are observed on different nights with similar seeing. If these techniques are not feasible, a theoretical SITF can be used.

From the object power spectrum $|O(u)|^{2}$ and the photon bias-compensated average LBT bispectrum $\sum_{t_{i}} I_{C}^{(3)}\left(u, v ; t_{i}\right)$ the object bispectrum $O^{(3)}(u, v)$ was obtained in the following way: (1) The modulus

$\left|O^{(3)}(u, v)\right|=|O(u)| \cdot|O(v)| \cdot|O(u+v)|$

of the object bispectrum $O^{(3)}(u, v)=\left|O^{(3)}(u, v)\right|$ $\exp [i \beta(u, v)]$ is derived from the object power spectrum. (2) The complex object bispectrum $O^{(3)}(u, v)$ is obtained by combining $\left|O^{(3)}(u, v)\right|$ from the previous step with the phasor

$\exp [i \beta(u, v)]=\frac{\sum_{t_{i}} I_{C}^{(3)}\left(u, v ; t_{i}\right)}{\left|\sum_{t_{i}} I_{C}^{(3)}\left(u, v ; t_{i}\right)\right|}$

of the photon bias-compensated average LBT bispectrum. This technique is possible as the phasor of the object bispectrum is identical to the phasor of the average bispectrum (Lohmann et al. 1983).

\subsection{Image reconstruction from the object bispectrum by the iterative building block method}

The diffraction-limited object intensity distribution $o(x)$ was reconstructed from the object bispectrum $O^{(3)}(u, v)$ by the iterative building block method (Hofmann \& Weigelt 1990; Hofmann \& Weigelt 1993; Reinheimer et al. 1993). The building block method searches for the diffraction-limited reconstruction which has the best agreement with the measured object bispectrum $O^{(3)}(u, v)$.

\section{Results of the LBT interferometry simulations}

\subsection{Laboratory simulation of LBT interferometry}

In our laboratory experiment the LBT interferograms were generated in the laboratory setup shown in Fig. 4. Atmospheric turbulence was generated by a heater. Small variations of the air temperature cause variations of the refractive index and therefore random wavefront degradations and speckle interferograms in the image plane. A mask simulating the LBT pupil function was inserted in front of a telescope lens. The speckle interferograms of various objects were recorded with a CCD camera in the focal plane.

Figure 5 shows the results of the LBT laboratory experiment. The details of the speckle masking interferometry image processing theory are described in Sect. 2. Figure 5a is the LBT pupil function. The geographic latitude $+30^{\circ}$ of the LBT site, an object declination of $+60^{\circ}$ and data recording at four different rotation angles of the earth during 12 hours were simulated (aperture synthesis by earth rotation). The $u v$-coverage of the experiment is shown in Fig. 5b. Figures 5c and 5d show the laboratory object. The brightness of the stars is $1.0,0.59,0.39$, and 0.13 . Figure $5 \mathrm{e}$ is one of only 200 recorded point source interferograms with simulated seeing corresponding to a Fried parameter $r_{0}=2 \mathrm{~m}$. For each of four chosen earth rotation angles, 50 point source interferograms and 100 object interferograms were recorded. Figure $5 \mathrm{f}$ is one of 400 recorded interferograms of the star cluster (degraded by speckle noise and CCD noise). Figures $5 \mathrm{~g}$ and $5 \mathrm{~h}$ show the diffraction-limited image reconstructed from the 400 interferograms by speckle masking and the building block method. 


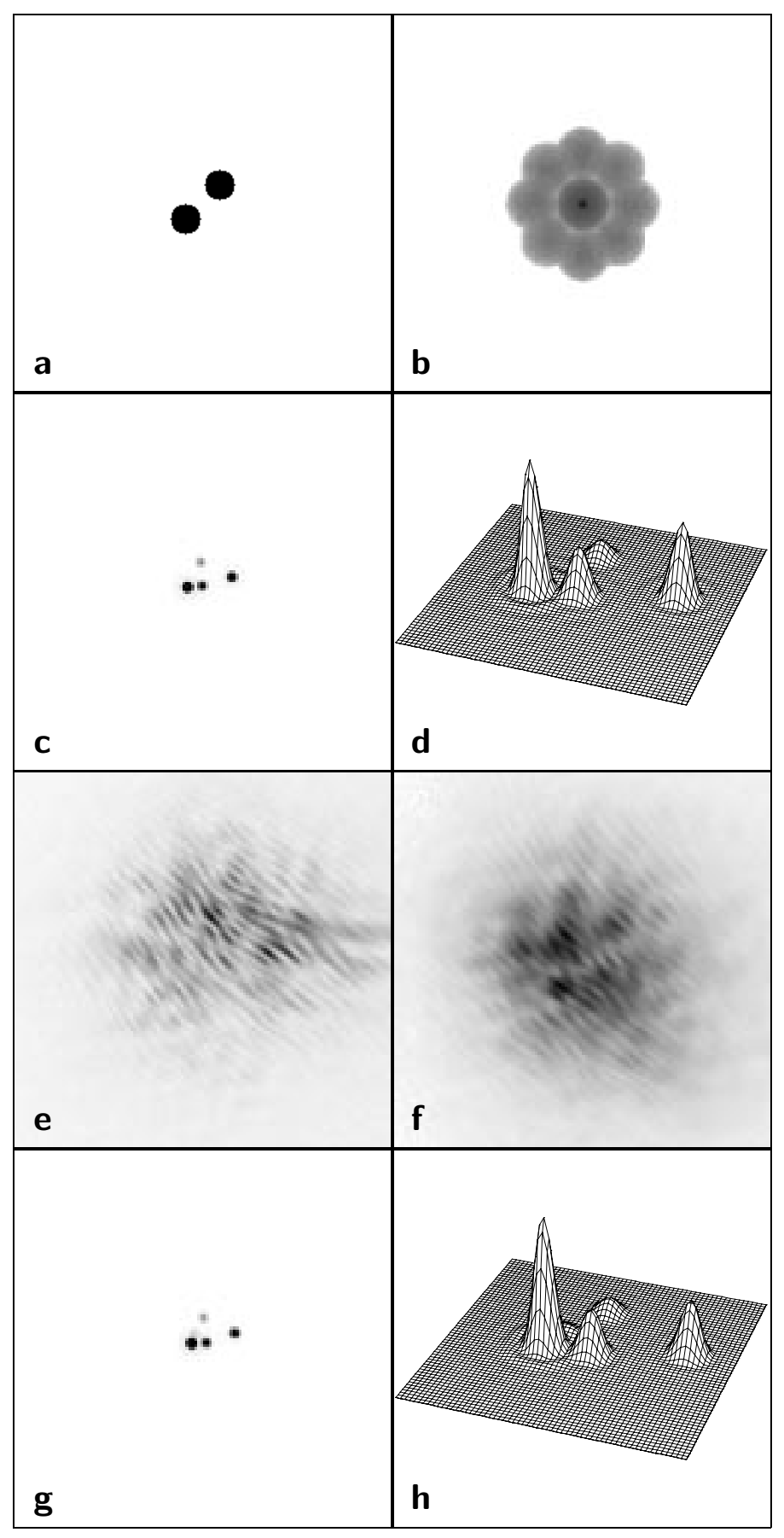

Fig. 5. Laboratory simulation of interferometric speckle masking imaging with the LBT: a) LBT pupil function, b) $u v$-coverage of the laboratory simulation (geographic latitude $\sim+30^{\circ}$; object declination $+60^{\circ}$; 12 -hour aperture synthesis time; data recording at four different earth rotation angles), c), d) object (star cluster), e) one of the 200 point source interferograms (required for calibrating the speckle transfer function) with simulated seeing corresponding to a Fried parameter $r_{0}=2 \mathrm{~m}$, f) one of the 400 LBT interferograms of the star cluster, g) and h) diffraction-limited image reconstructed from 400 interferograms by speckle masking and the building block method (1000 iterations) 


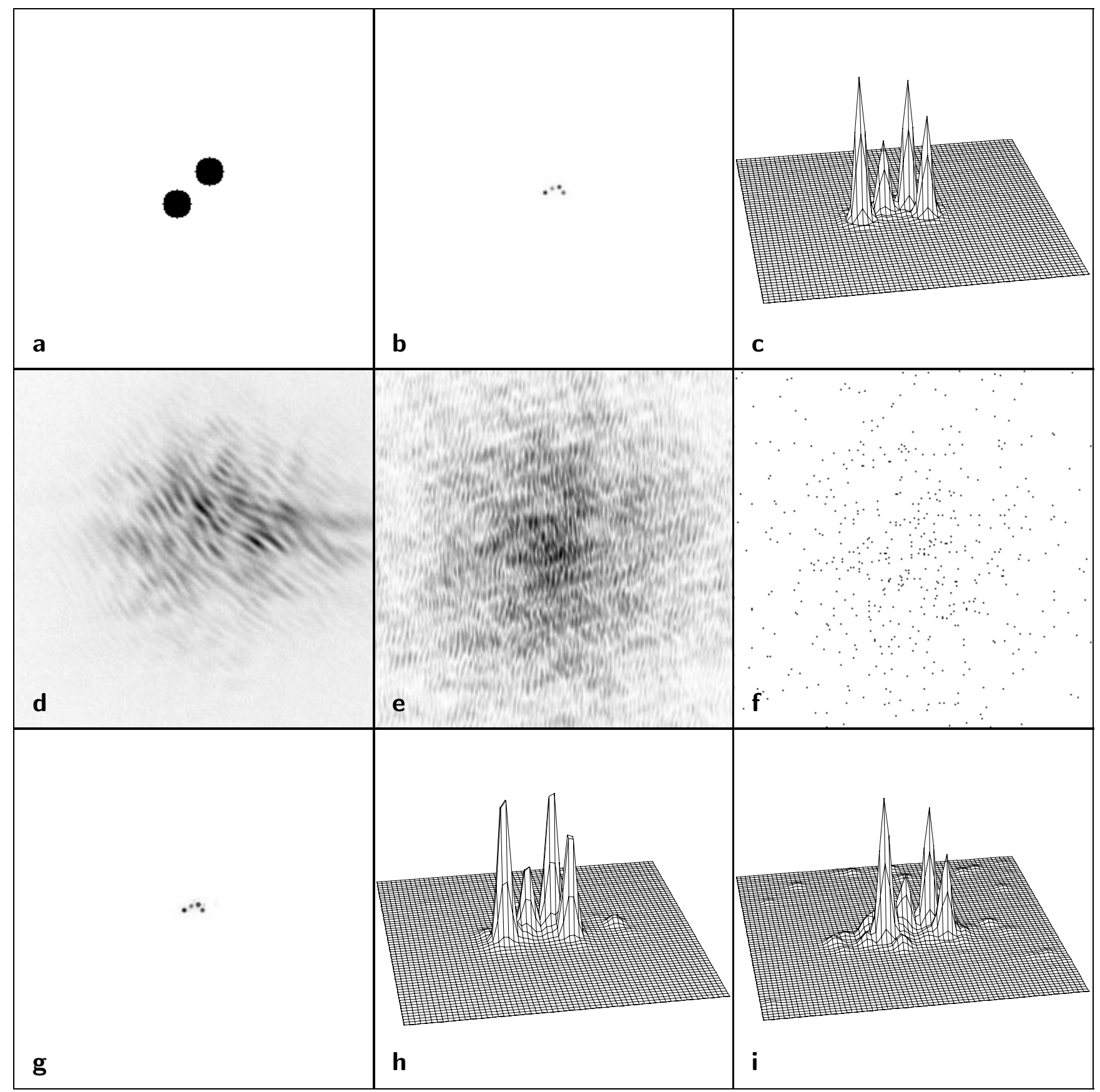

Fig. 6. Computer simulation of speckle masking interferometry with the LBT at optical wavelengths (Fried parameter $r_{0}=40$ cm, corresponding to 0.35 arcsec seeing): a) LBT pupil function, b, c) object (star cluster), d) one of the 200000 generated point source interferograms (for the calibration of the speckle transfer function) with simulated seeing corresponding to a Fried parameter $r_{0}=40 \mathrm{~cm}, \mathbf{e}$ ) one of the 200000 generated object interferograms before simulation of photon noise, f) one of the 200000 generated LBT speckle interferograms of the star cluster after injection of photon noise corresponding to a mean count number of 500 photoevents per frame, g, h) diffraction-limited image reconstructed from the 200000 photon noise-degraded interferograms by speckle masking and the building block method ( 500 photoevents/interferogram; 5000 iterations of the building block method; star separation $\sim 13$ milli-arcsec for $\lambda=5500 \AA$ and LBT diameter $22 \mathrm{~m}$ ), and i) diffraction-limited image reconstructed from the 200000 interferograms degraded by photon noise corresponding to a mean count number of only 200 photoevents/interferogram. The $u v$-coverage of the computer experiment (geographic latitude $+30^{\circ}$; object declination $+60^{\circ}$; 12-hour aperture synthesis; data recording at 71 different rotation angles of the earth) is shown in Fig. 8b 


\subsection{Results of LBT computer simulations}

Figures 6 and 8 show the results of our LBT computer experiments with a star cluster and an extended object. The image processing theory is described in Sect. 2. The reconstructions have exact diffraction-limited resolution, for example 6.1 milli-arcsec resolution for $\lambda=5500 \AA$ and $22 \mathrm{~m}$ interferometer diameter.

\subsubsection{Star cluster simulation}

Figure 6 shows a computer simulation of interferometric LBT imaging of a star cluster. Figure $6 \mathrm{a}$ is the LBT pupil function of the experiment. In this experiment an object declination of $+60^{\circ}$, a geographic latitude of the LBT site of $+30^{\circ}$, and data recording at 71 different rotation angles of the earth during a time period of 12 hours were simulated (aperture synthesis by earth rotation). Figures $6 \mathrm{~b}$ and $6 \mathrm{c}$ are the object. The brightness of the stars from left to right is $1.0,0.53,0.92$, and 0.72 . The smallest simulated separation is $\sim 13$ mas (for $\lambda=550 \mathrm{~nm}$ and $22 \mathrm{~m}$ baseline). Figure $6 \mathrm{~d}$ is one of the 200000 generated point source LBT interferograms with simulated seeing corresponding to a Fried parameter $r_{0}=40 \mathrm{~cm}$, corresponding to 0.35 arcsec seeing. For each of the 71 chosen earth rotation angles, 2817 interferograms were computed.

Figure 6e is one of the 200000 generated LBT interferograms of the star cluster. Figure $6 \mathrm{f}$ shows the same object interferogram after injection of photon noise corresponding to a mean count number of 500 photoevents per frame. Figures $6 \mathrm{~g}$ and $6 \mathrm{~h}$ show the diffraction-limited image reconstructed from the 200000 noise-degraded interferograms consisting of 500 photoevents/interferogram by speckle masking and the building block method. Figure $6 \mathrm{i}$ is the diffraction-limited image reconstructed from 200000 noise-degraded interferograms consisting of 200 photoevents/interferogram. 200000 interferograms correspond to only 1.1 hours of data recording time for a frame rate of 50 frames per second. Table 1 summarizes the parameters of the star cluster experiment and the photometric error of the reconstructions.

200 photoevents per interferogram correspond to a total magnitude $\sim 14.3$ for two $8 \mathrm{~m}$ telescopes, $20 \mathrm{msec}$ exposure time per interferogram, $5 \mathrm{~nm}$ filter bandwidth, and $10 \%$ quantum efficiency of detector plus optics (see Fig. 7). With the above-mentioned intensity ratios of the four stars we obtain for the individual four stars the simulated magnitudes of 15.6, 15.8, 16.4, and 17.1.

\subsubsection{Simulations of an extended object}

Figure 8 shows the results of a computer simulation with an extended object. In this experiment each photon noisedegraded object interferogram consisted of 2000 photoevents. All other experimental parameters were identical as in the star cluster experiment. The experimental parameters are summarized in Table 1 . The average photo-

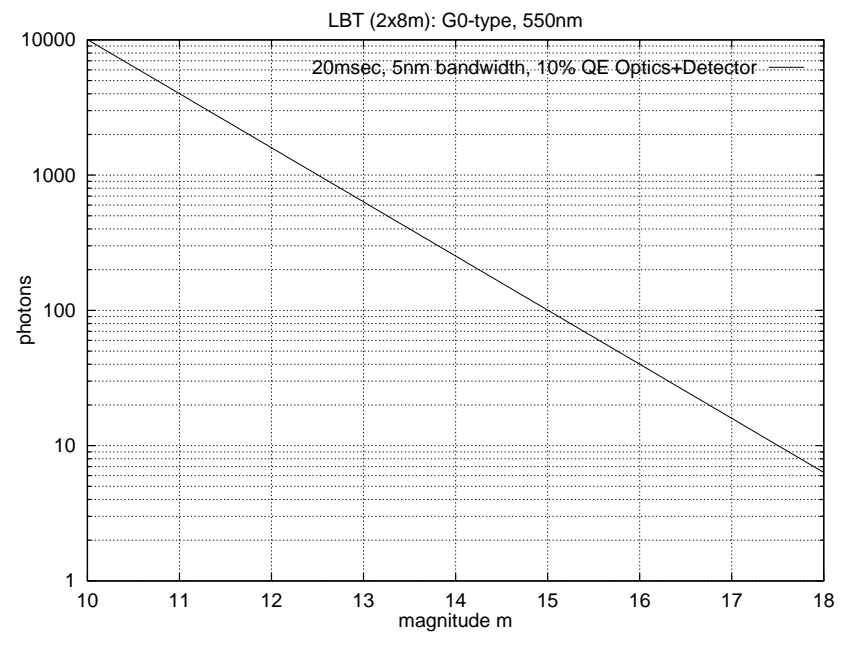

Fig. 7. Number of photons detected in LBT interferograms plotted against $V$-magnitude of a G0 star for $20 \mathrm{msec}$ exposure time/frame, $5 \mathrm{~nm}$ spectral bandwidth (FWHM; use of only one spectral channel), and $10 \%$ quantum efficiency of optics and detector. The flux of a G0-type star was taken from Allen (1973)

metric error of the reconstruction (Fig. 8i) is about $20 \%$. 2000 photoevents correspond to a total magnitude of 11.3 for two $8 \mathrm{~m}$ telescopes, $20 \mathrm{msec}$ exposure time per interferogram, $5 \mathrm{~nm}$ filter bandwidth, and $10 \%$ quantum efficiency of detector plus optics. Since the object consists of about 10 diffraction-limited resolution elements of similar brightness, we obtain for the brightness of the brightest resolution element (nucleus) about magnitude 13 and for the faintest about magnitude 14. Much fainter objects can be reconstructed if observing time is longer than 1.1 hours, if seeing can be improved by partial adaptive optics, or if data can simultaneously be recorded in, for example, 10 to 100 spectral channels of $5 \mathrm{~nm}$ bandwidths.

\section{Conclusions}

We have presented a method for interferometric imaging with the Large Binocular Telescope. The laboratory and computer experiments demonstrate that image reconstruction from LBT interferograms can easily be performed by the speckle masking method combined with the iterative building block method. The experimental results show the dependence of the reconstructed images on photon noise. The smallest simulated number of photoevents per interferogram was 200. This photoevent number corresponds to magnitude $\sim 14.3$ for two $8 \mathrm{~m}$ telescopes, $20 \mathrm{msec}$ exposure time per interferogram, $5 \mathrm{~nm}$ filter bandwidth, and $10 \%$ quantum efficiency of detector plus optics. 200000 interferograms were evaluated per experiment corresponding to only 1.1 hours of data recording time for a frame rate of 50 frames per sec. The mean photometric error of the reconstructed stars of the $14.3^{\mathrm{m}}$ 


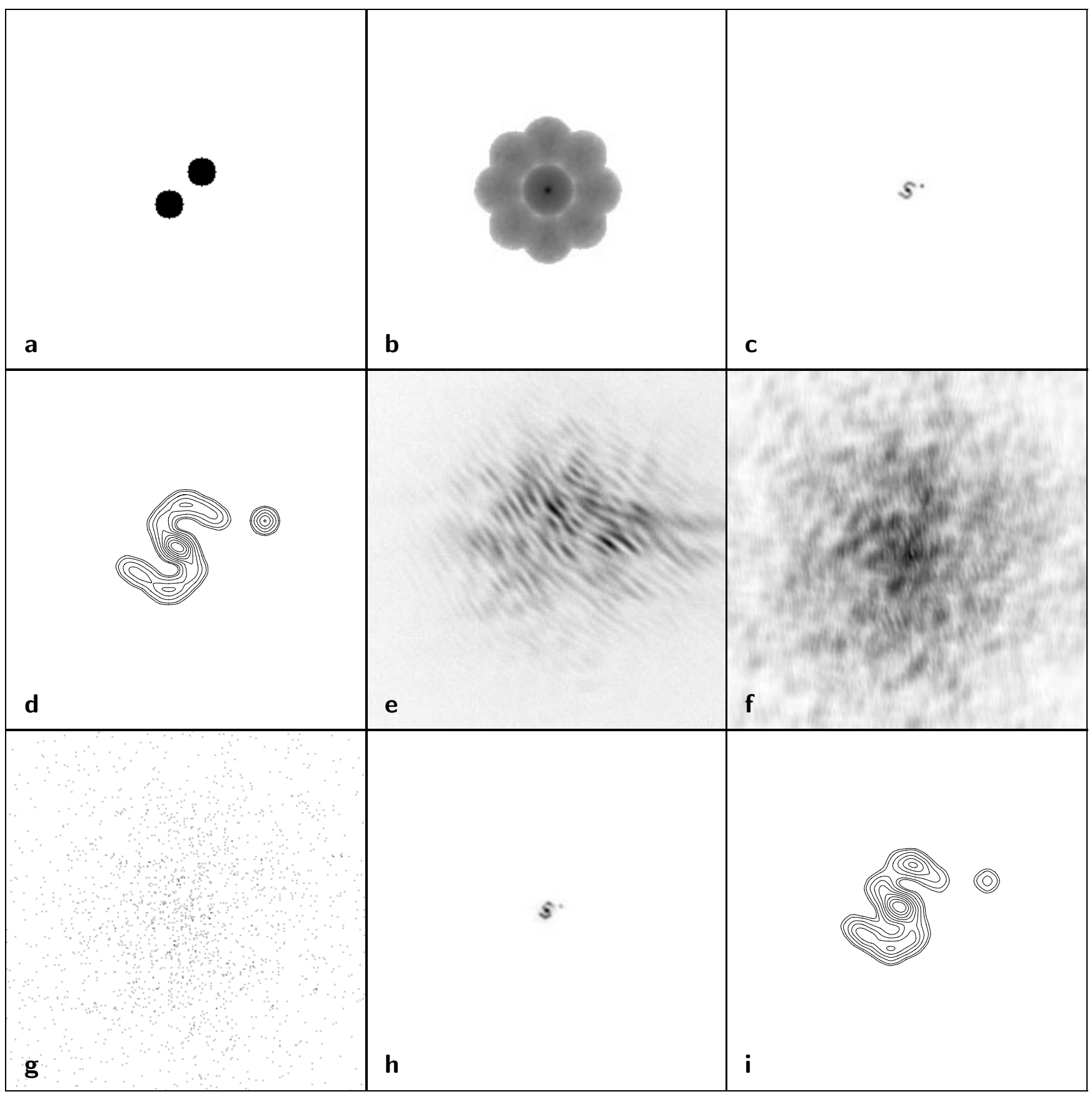

Fig. 8. Computer simulation of speckle masking interferometry of an extended object (Fried parameter $r_{0}=40 \mathrm{~cm}$, corresponding to 0.35 arcsec seeing): a) LBT pupil function, b) $u v$-coverage of the computer experiment (geographic latitude $+30^{\circ}$; object declination $+60^{\circ}$; 12 -hour aperture synthesis; data recording at 71 different rotation angles of the earth), $\left.\mathbf{c}, \mathbf{d}\right)$ object (galaxy), e) one of the generated LBT point source speckle interferograms, f) one of the 200000 generated object interferograms before simulation of photon noise, g) one of the generated 200000 LBT speckle interferograms of the object after injection of photon noise corresponding to a mean count number of 2000 photoevents per interferogram, and $\mathbf{h}$, i) the diffraction-limited image reconstructed from the 200000 photon noise-degraded interferograms by speckle masking and the building block method (2000 photoevents/interferogram; 6000 iterations of the building block method; galaxy diameter $\sim 44$ milli-arcsec for $\lambda=5500 \AA$ and LBT diameter $22 \mathrm{~m}$ ). The contour levels are 15, 20,30,40,50,60,70, 80, and 90\% of the peak intensity (in order to show greater detail, Figs. d and i have been enlarged) 
Table 1. Parameters of the computer experiments shown in Figs. 6 and 8. A frame rate of 50 frames/sec was assumed for the calculation of the data recording times. The field of view of the interferograms and the reconstructions is $\sim 0.69^{\prime \prime} \times 0.69^{\prime \prime}=256 \times 256$ pixels (pixel size $\sim 2.710^{-3}$ arcsec)

\begin{tabular}{lrr}
\hline Experimental parameters & Star cluster & Extended object \\
\hline Figure number & 6 & 8 \\
Geographic latitude & $+30^{\circ}$ & $+30^{\circ}$ \\
Declination & $+60^{\circ}$ & $+60^{\circ}$ \\
Number of earth rotation angles & 71 & 71 \\
Aperture synthesis time & $12 \mathrm{~h}$ & $12 \mathrm{~h}$ \\
Number of interferograms per earth rotation angle & 2817 & 2817 \\
Data recording time per angle & $\sim 57 \mathrm{~s}$ & $\sim 57 \mathrm{~s}$ \\
Total number of interferograms & 200000 & 200000 \\
Total data recording time & $\sim 1.1 \mathrm{~h}$ & $1.1 \mathrm{~h}$ \\
Fried parameter $r_{0}$ & $40 \mathrm{~cm}$ & $40 \mathrm{~cm}$ \\
Number of building block iterations & 5000 & 6000 \\
Photoevents per interferogram; mean intensity error & 500 events; $6.9 \%$ & $2000 ; 20 \%$ \\
Photoevents per interferogram; mean intensity error & 200 events; $9.5 \%$ & \\
\hline
\end{tabular}

star cluster is about $9.5 \%$ (for 200000 interferograms, $r_{0}=$ $40 \mathrm{~cm}, 200$ photoevents/interferogram, only one spectral channel of $5 \mathrm{~nm}$ bandwidth). The simulated brightness of the individual four stars is $15.6,15.8,16.4$, and 17.1. Objects of about 18th magnitude and fainter can be studied with 6 mas resolution if observing time is increased, data are simultaneously recorded in many spectral channels, and if partial wavefront compensation is achieved by an artificial laser guide star system.

\section{References}

Allen C.W., 1973, in: Astrophys. Quant. The Athlone Press, London, p. 207

Foy R., Labeyrie A., 1985, A\&A 152, L29

Fugate R.Q., Fried D.L., Ameer G.A., et al., 1991, Nat 353, 144

Goodman J.W., 1985, in: Statistical Opt. Wiley \& Sons, New York, Chapter 9.6

Hofmann K.-H., Weigelt G., 1990, Image Reconstruction from the Bispectrum Using an Iterative Algorithm, Applications of the Method to Astronomical Objects, in: Digital Image Synthesis and Inverse Optics, 9-13 July 1990. In: Gmitro A.F., Idell P.S., LaHaie I.J. (eds.), Soc. Photo-Opt. Instr. Eng. 1351, 522

Hofmann K.-H., Weigelt G., 1993, A\&A 278, 328
Labeyrie A., 1970, A\&A 6, 85

Lohmann A.W., Weigelt G., Wirnitzer B., 1983, Appl. Opt. 22,4028

McCarthy D.W. Jr., Hege E.K., Freeman J.D., et al., 1988, Interferometry with the Columbus Telescope: Design Considerations Based on MMT Experience and Imaging Simulations, in: Very Large Telescopes and their Instrumentation, 21-24 March, 1988, Ulrich M.-H. (ed.), European Southern Observatory, Garching, Germany, p. 787

Pehlemann E., Hofmann K.-H., Weigelt G., 1992, A\&A 256, 701

Primmerman C.A., Murphy D.V., Page D.A., Zollars B.G., Barclay H.T., 1991, Nat 353, 141

Reinheimer T., Weigelt G., 1990, Optical Long-Baseline Interferometry in Astronomy, in: Optics in Complex Systems, 5-10 August, 1990, Lanzl F., Preuss H.-J., Weigelt G. (eds.), Soc. Photo-Opt. Instr. Eng. 1319, 678

Reinheimer T., Hofmann K.-H., Weigelt G., 1993, A\&A 279, 322

Strittmatter P.A., 1988, Columbus Project Overview, in: Very Large Telescopes and their Instrumentation, 21-24 March 1988, Ulrich M.-H. (ed.), European Southern Observatory, Garching, Germany, p. 29

Weigelt G., 1977, Opt. Commun. 21, 55

Weigelt G., Wirnitzer B., 1983, Opt. Lett. 8, 389

Wirnitzer B., 1985, J. Opt. Soc. Am. A 2, 14 